\title{
PERSISTENT CORONAVIRUS INFECTION OF PROGENITOR OLIGODENDROCYTES
}

\author{
Yin Liu and Xuming Zhang*
}

\section{INTRODUCTION}

Mouse hepatitis virus (MHV) is a prototype of murine coronavirus. It can infect rodents and causes enteritis, hepatitis, and central nervous system (CNS) diseases. Infection of mouse CNS with neurovirulent MHV strains usually results in acute encephalitis followed by demyelination. ${ }^{1,2}$ If the majority of the virus can be cleared from the CNS, encephalitis then resolves; if mice survive the acute phase, demyelination develops. Although acute demyelination can be detected histologically as early as 6 days postinfection (p.i.), extensive demyelination is often not seen until 4 weeks p.i. ${ }^{3}$ However, infectious virus can no longer be isolated from the CNS at this time, although viral RNAs continue to persist in the CNS for more than one year, during which time period demyelination is concomitantly detectable. ${ }^{4,5}$ The correlation between viral RNA persistence and demyelination in the CNS suggests that viral persistence may be a prerequisite for the development of CNS demyelination. However, virtually nothing is known as to how viral persistence contributes to demyelination.

Previous studies attempted to establish an in vitro system of glial or fibroblast cell culture for viral persistence. ${ }^{6,7}$ Unfortunately, the persistent infection established in these cells is productive, i.e., generation of infectious viruses with significant virus titers. This type of persistence does not reflect on the infection of animal CNS. Recently we established a persistent MHV infection in a progenitor rat oligodendrocyte. We showed that MHV RNAs were continuously detected in infected cells of more than 20 passages. However, no infectious virus could be isolated from these cells. This phenomenon resembles the persistent, nonproductive infection in animal CNS. To understand the molecular basis of viral persistence in host cells, we analyzed the gene expression profiles of the persistently infected cells by using DNA microarray technology and RTPCR. We found that the expression of a substantial number of cellular genes was altered by viral persistence. Interestingly, although persistently infected progenitor cells could be induced to differentiate into mature oligodendrocytes, the number of dendrites and level of myelin basic protein were markedly reduced in persistent cells. This finding indicates

\footnotetext{
* University of Arkansas for Medical Sciences, Little Rock, Arkansas 72205.
} 
that MHV persistence has an inhibitory effect on oligodendrocyte differentiation and dendrite outgrowth and provides the first direct evidence linking viral persistence to demyelination.

\section{MATERIALS AND METHODS}

\subsection{Cell, Virus, and Reagents}

The CG (central glial)-4 cell is a permanent, undifferentiated type 2 oligodendrocyte/ astrocyte progenitor cell that was established during a primary neural cell culture derived from the brain of newborn Sprague-Dawley rat pups (1-3 days postnatal). ${ }^{8}$ CG-4 cell culture was maintained as described previously. ${ }^{9}$ MHV strain JHM was obtained from Michael Lai's laboratory. It was propagated in mouse astrocytoma cell line DBT cells and was used throughout this study. Virus titers were determined by plaque assay as described previously.

\subsection{Reverse Transcription-Polymerase Chain Reaction (RT-PCR)}

The intracellular RNAs were reverse-transcribed into cDNAs by using a random hexomer oligonucleotide primer (Invitrogen, Inc.), and the cDNAs were amplified by PCR using gene specific primers as described previously. ${ }^{10}$ The following gene-specific primer pairs were used in PCR: 5'BamN (5'-TAG GGA TCC ATG TCT TTT GTT CCT3') and 3'EcoN515 (5'-TAG GAA TTC GGC AGA GGT CCT AG-3') for viral nucleocapsid (N) gene; 5'-cmyc (5'-TTT CTC GAG GCC ACG ATG CCC CTC AAC GTG AGC TTC-3') and 3'-cmyc (5'-TTT GAA TTC CCA GAG TCG CTG CTG GTG GTG GGC-3') for c-myc gene; 5'-sox (5'-TTT CTC GAG ATG GTG CAG CAG GCC GAG AGC-3') and 3'-sox (5'-TTG AAT TCC ATA CGT GAA CAC CAG GTC GGA3') for Sox11 gene; 5'-bcl2 (5'-TTT CTC GAG GCC ACC ATG GCG CAC GAT GGG AGA ACA-3') and 3'-bcl2 (5'-TTT GAA TTC CCT TGT GGC CCA GAT AGG CAC CCA-3') for Bcl-2 gene; 5' mb-actin (5'-ACC AAC TGG GAC GAT ATG GAG AAT A3') and 3'mb-actin (5'-TAC GAC CAG AGG CAT ACA GGG ACA-3') for $\beta$-actin, which was used as an internal control.

\subsection{DNA Microarray Analysis}

For DNA microarray analysis, mRNAs were extracted from persistent- or mockinfected CG-4 cells at passage 20 p.i. using the Qiagen RNAeasy kit according to the manufacturer's instructions. The purity and quantity of the RNAs were determined by spectrophotometry. The levels of individual mRNA species were determined by microarray using the Affymetrix Oligo Gene Chip (U34), which detects approximately 7,000 known genes and 1,000 EST clusters. The DNA microarray analysis was carried out at the University of Iowa DNA Core facility. A 2-fold or greater difference between the test (persistently infected CG- 4 cells) and the control (mock-infected CG- 4 cells) was considered a significant change while any genes that are absence (below detectable level) in both test and control cells were excluded from the analysis. 


\subsection{Western Blot Analysis}

Western blot analysis was carried out as described. ${ }^{9}$ The antibodies used in this study include a polyclonal rabbit antibody specific to Bcl-2 $(0.2 \mu \mathrm{g} / \mathrm{ml})$ (Cell Signals, Inc.), a monoclonal antibody $(\mathrm{mAb})$ to rat myelin basic protein (MBP) $(1 \mu \mathrm{g} / \mathrm{ml})$ (Chemicon Internation, Inc.), and a mAb to $\beta$-actin (1:5000) (Sigma).

\subsection{Plasmid Construction, DNA Transfection, and Selection of Stable Transfectants}

The Bcl-2 gene was kindly provided by Marie Hardwick (Johns Hopkins University) and was subcloned into pcDNA3, resulting in pcDNA3/Bcl-2. CG-4 cells were transfected with pcDNA3/Bcl-2 DNA with FuGene-6 transfection reagent (Roche), and selected for stable expression of Bcl-2 with G418 and by Western blot analysis.

\subsection{Assay for Cell Viability}

Trypan blue was used for staining dead cells following MHV infection or mockinfection. The dead cells were counted in 3 independent experiments.

\subsection{Immunofluorescence Staining}

The immunocytochemistry method used in this study was previously described. ${ }^{9}$ Stained cells were observed under a fluorescent microscope (Olympus IX70) and photographs were taken with an attached digital camera (MagnaFire).

\section{RESULTS AND DISCUSSION}

\subsection{Establishment of Persistent, Nonproductive MHV Infection in Rat Progenitor Oligodendrocytes}

In a recent study, we reported that a brief treatment of CG-4 cells with fetal bovine serum rescued cells from MHV killing. ${ }^{10}$ To extend these observations, we collected cells at various passages and determined the presence of viral genomic RNA by RT-PCR and the infectious virus by plaque assay. The viral genomic RNA at the $\mathrm{N}$ gene locus was consistently detected by RT-PCR throughout all 20 passages. The identity of the RT-PCR fragments was confirmed by DNA sequencing. However, no infectious virus could be detected from these cells beyond passage 3 . This result demonstrates that viral RNA was able to persist in CG-4 cells without the production of infectious virus. This phenomenon resembles MHV persistence in the animal CNS. The establishment of MHV RNA persistence in glial cells provides a useful system for studying the molecular mechanisms of viral persistence and demyelination. 
Table 1. Genes that are upregulated in MHV-persistent CG-4 cells.

\begin{tabular}{lllll}
\hline \multicolumn{1}{c}{ Category } & \multicolumn{1}{c}{ Gene name } & Acces. no. & Fold( $\uparrow)$ & \multicolumn{1}{c}{ Function } \\
\hline $\begin{array}{l}\text { MARK/ } \\
\text { phosphatase }\end{array}$ & $\begin{array}{l}\text { Dual specificity } \\
\text { phosphatase(MKP3) }\end{array}$ & X94185 & 29.9 & $\begin{array}{l}\text { Inhibits FGF/RAS/ } \\
\text { MAPK pathway }\end{array}$ \\
$\begin{array}{l}\text { Cell cycle/ } \\
\text { apoptosis }\end{array}$ & Bcl-2 & L14680 & 26 & $\begin{array}{l}\text { Anti-apoptotic, cell } \\
\text { survival }\end{array}$ \\
$\begin{array}{l}\text { Tanscription } \\
\text { /oncogene }\end{array}$ & c-Myc & Y00396 & 9.8 & $\begin{array}{l}\text { Oncogenic, cell } \\
\text { survival/apoptosis }\end{array}$ \\
$\begin{array}{l}\text { Neural/glial } \\
\text { cell-specific }\end{array}$ & $\begin{array}{l}\text { Sry-related HMG- } \\
\text { box protein Sox11 }\end{array}$ & AJ004858 & 3.2 & $\begin{array}{l}\text { Inhibits oligo.matur./ } \\
\text { myelin expression }\end{array}$ \\
\hline
\end{tabular}

\subsection{Viral RNA Persistence Altered the Gene Expression Profile of Progenitor Oligodendrocytes}

To determine whether viral RNA persistence in progenitor oligodendrocytes alters the cellular gene expression, we used DNA microarray technology to compare the mRNA levels in persistently infected CG-4 cells with those of mock-infected CG-4 cells. Overall, approximately 350 genes were significantly upregulated, whereas only about 30 genes were downregulated in persistently infected CG-4 cells as compared with those of mock-infected CG-4 cells at passage 20. Most notable among the differentially expressed genes are those that are involved in signal transduction, cell cycle, cell survival and death, and differentiation. For examples, the expression of cellular oncogenes Bcl-2 and c-myc increased by 26 and 9.6 fold, respectively; the Sry-related HMG-box protein Sox 11 was increased by 3.2 fold (Table 1). To confirm the DNA microarray results, we selected a few genes for analysis with RT-PCR. Indeed, a significant increase of the Sox11, c-myc, and Bcl-2 mRNAs was detected at passage 20 in persistently infected CG-4 cells as compared to those in mock-infected CG-4 cells (Figure 1). These results suggest that persistent infection might have altered many biological properties of CG-4 cells, such as cell proliferation and differentiation. Significantly, because Sox 11 is a transcription factor, a negative regulator of transcription of many myelin-associated proteins, the increase of Sox11 expression might have a negative consequence on myelination by persistently infected oligodendrocytes.
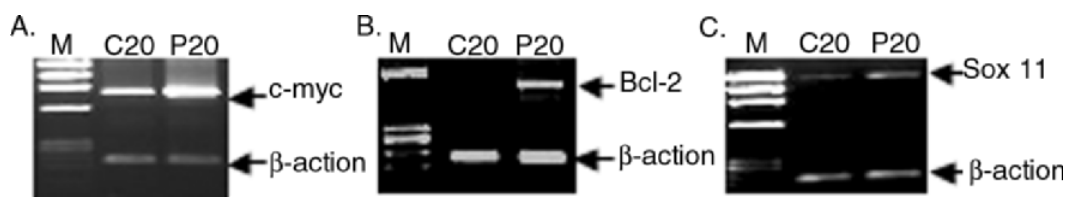

Figure 1. RT-PCR detection of mRNAs for c-myc (A), Bcl-2 (B), and Sox 11 (C) genes in MHV persistently infected CG-4 cells. Intracellular RNAs were isolated from mock-infected cells as a control (lane C) or from MHV-persistently infected CG-4 cells at passage 20 (lane P20) and were detected by RT-PCR with gene specific primers. B-actin gene was used as an internal control. M, molecular size marker. 


\subsection{Role of Bcl-2 in the Establishment and Maintenance of Viral RNA Persistence in CG-4 Cells}

To determine whether the change in host cellular gene expression also plays a role in the establishment of viral persistence in CG-4 cells, we chose the Bcl-2 gene as an example. Because Bcl-2 is an anti-apoptotic protein of the mitochondria, its increased expression by viral persistence must have an effect on cell survival. To test this hypothesis, we stably expressed the Bcl-2 gene in CG-4 cells. Overexpression of Bcl-2 in stable expressing CG-4 cells was confirmed by Western blot analysis (data not shown). When cells were infected with MHV, the extent of apoptosis in Bcl-2- expressing cells was significantly lower than in cells expressing the vector alone. This result suggests that upregulation of Bcl-2 by viral persistence may have beneficial effect on cell survival, regardless of whether the upregulation of Bcl-2 is the result of host response to viral infection or is the direct effect of virus infection. It is conceivable that the survival of host cells is also essential for the virus to persist. This finding may also provide a potential mechanism for the coexistence of the host and the parasite and represent a close relationship between the host cell and the persistence of viral RNA.

To determine whether and to what extent surviving cells still harbored virus, cells from various passages were collected and intracellular RNAs were isolated. The presence of the viral genome was determined by RT-PCR with primers specific to MHV N gene, and the production of infectious virus was measured by plaque assay in DBT cells. Indeed, the $\mathrm{N}$ gene region of the infecting viral genome was detected in all 10 passages, indicating the establishment of persistent infection. In contrast, infectious virus could be isolated only in the first 2 passages. These results suggest that cellular anti-apoptotic gene Bcl-2 may play a role in the establishment and maintenance of MHV persistence in CNS cells. By extrapolating the findings from Bcl-2, it is attempting to suggest that numerous cellular genes that are related to cell cycle, growth, proliferation, and survival and that are altered by viral persistence may play a vital role in the process of viral RNA persistence in CNS cells.

\subsection{Effect of MHV Persistence on Differentiation of Progenitor CG-4 Cells}

Because the destruction of the myelin sheath surrounding neuron axons in the CNS is the hallmark of demyelination in persistently MHV-infected animals and in multiple sclerosis patients, we further determined the ability of MHV-persistently-infected progenitor CG-4 cells to differentiate into mature oligodendrocytes and to form dendrites. The myelin basic protein, a marker expressed during the late stage of differentiation, was used to identify the morphology of the mature oligodendrocyte. Both persistently infected and mock-infected CG-4 cells were cultured under the condition that allows the cells to differentiate into mature oligodendrocyte. Although both cells could differentiate into mature oligodendrocytes, the oligodendrocytes differentiated from MHV-persistently infected CG-4 cells at both passage 20 and passage 30 had either fewer dendrites or less branched dendrites as compared with those derived from mock-infected CG-4 (data not shown). This result indicates that MHV persistence has an inhibitory effect on oligodendrocyte differentiation and dendrite formation. Consistent with this result is the finding that the expression of the myelin-basic protein was also reduced in persistently infected oligodendrocytes (data not shown). This study provides the first direct evidence 
that links MHV RNA persistence to malfunction of affected oligodendrocytes in dendrite (and possibly in vivo myelin) formation.

\section{ACKNOWLEDGMENTS}

This work was supported by grants from the National Institutes of Health (AI 47188 and NS 47499). We thank Paul Drew (UAMS) for kindly providing the CG-4 cells, Stephen Stohlman (Keck School of Medicine, University of Southern California, Los Angeles) for the N monoclonal antibody, and Marie Hardwick (Johns Hopkins University School of Medicine, Baltimore) for the Bcl-2 gene.

\section{REFERENCES}

1. Weiner, L. P., 1973, Pathogenesis of demyelination induced by a mouse hepatitis virus (JHM virus), Arch Neurol. 28:67-74.

2. Fleury, H. J., Sheppard, R. D., Bornstein, M. B., and Raine, C. S., 1980, Further ultrastructural observations of virus morphogenesis and myelin pathology in JHM virus encephalomyelitis, Neuropathol. Appl. Neurobiol. 6:165-179.

3. Lavi, E., Gilden, D. H., Highkin, M. K., and Weiss, S. R., 1984, Persistence of mouse hepatitis virus A59 RNA in a slow virus demyelinating infection in mice as detected by in situ hybridization, J. Virol. 51:563-566.

4. Knobler, R. L., Lampert, P. W., and Oldstone, M. B., 1982, Virus persistence and recurring demyelination produced by a temperature-sensitive mutant of MHV-4, Nature 298:279-280.

5. Bergmann, C., Dimacali, E., Stohl, S., Wei, W., Lai, M. M. C., Tahara, S., and Marten, N., 1998, Variability of persisting MHV RNA sequences constituting immune and replication-relevant domains, Virology 244:563-572.

6. Sawicki, S. G., Lu, J. H., and Holmes, K. V., 1995, Persistent infection of cultured cells with mouse hepatitis virus (MHV) results from the epigenetic expression of the MHV receptor, J. Virol. 69:5535-5543.

7. Chen, W., and Baric, R. S., 1996, Molecular anatomy of mouse hepatitis virus persistence:coevolution of increased host cell resistance and virus virulence, J. Virol. 70:3947-3960.

8. Louis, J. C., Magal, E., Muir, D., Manthorpe, M., and Varon, S., 1992, CG-4, a new bipotential glial cell line from rat brain, is capable of differentiating in vitro into either mature oligodendrocytes or type-2 astrocytes, J. Neurosci. Res. 31:193-204.

9. Liu, Y., Cai, Y., and Zhang, X., 2003, Induction of caspase-dependent apoptosis in cultured rat oligodendrocytes by murine coronavirus is mediated during cell entry and does not require virus replication, J. Virol. 77:11952-11963.

10. Liu, Y., and Zhang, X., 2005, Expression of cellular oncogene Bcl-xL prevents coronavirus-induced cell death and converts acute infection to persistent infection in progenitor rat oligodendrocytes, J. Virol. 79:47-56. 